\title{
Quality of Life Measurements after Cochlear Implantation
}

\author{
Christoph Loeffler, ${ }^{*}$, Antje Aschendorff ${ }^{1,2}$, Thorsten Burger ${ }^{2}$, Stefanie Kroeger ${ }^{2}$, Roland Laszig ${ }^{1,2}$ \\ and Susan Arndt ${ }^{1,2}$
}

\author{
${ }^{I}$ Department of Otorhinolaryngology, Head and Neck Surgery; University Medical Center Freiburg, Germany \\ ${ }^{2}$ Implant Center Freiburg within the Department of Otorhinolaryngology, Head and Neck Surgery; University Medical \\ Center Freiburg, Germany
}

\begin{abstract}
Cochlear implantation has a remarkable impact on the social life, activities and self-esteem of each patient. In order to objectify this comprehensive effect the term quality of life has been introduced. Various questionnaires have been developed to assess the quality of life in such patients.

In a review of the literature several generic and disease specific quality of life questionnaires were analyzed regarding the effect of cochlear implantation and changes over time after implantation.

Numerous disease specific instruments show a considerable improvement in total scores and in several subcategories, but
some generic instruments may not be sensitive enough to detect changes after cochlear implantation.

Nevertheless a respectable number of sensitive and validated instruments are available for use in these patients. These instruments recognize substantial improvements after cochlear implantation in several aspects of quality of life including physical, social and psychological domains.

Since quality of life questionnaires allow a comprehensive insight into patients' daily life and activities, quality of life measurements are an essential addition to speech perception tests to quantify the outcome after implantation. The measurement of cochlear implant related quality of life states an important instrument to provide information about the outcome of technical improvements, different treatment and rehab strategies in future.
\end{abstract}

Keywords: Sensineural hearing loss, Nijmegen cochlear implant questionnaire, postlingual deafness, speech perception.

\section{INTRODUCTION}

Cochlear implantation is a well established method for the treatment of deaf and profoundly hearing-impaired patients due to sensorineural hearing loss [1]. The improvement of hearing in terms of purely audiological performance is remarkable: audiological tests including monosyllabic word and sentence tests show a noticeable increase in open-word recognition after implantation of Cochlear implants $[2,3]$.

Hence, most clinical trials have set the focus on the improvement of the audiological performance.

But cochlear implantation affects not only hearing abilities, speech perception and speech production, it also has an outstanding impact on the social life, activities and self-esteem of each patient.

To measure and objectify this comprehensive effect the term health-related quality of life (QoL) has been defined. During the past there has been a growing interest in the evaluation of QoL. QoL scores are used to detect the benefit of different types of medical treatment [4].

*Address correspondence to this author at the Department of Otorhinolaryngology, Head and Neck Surgery, University Medical Center Freiburg, Killianstrasse 5, D-79106 Freiburg, Germany; Tel: +49 761270 4201; Fax: +49 761270 4075;

E-mail: christoph.loeffler@uniklinik-freiburg.de
Although multiple definitions of QoL exist [5] it is usually utilized as patients' emotional, social and physical well-being, including their ability to function in the ordinary tasks of living. The vague definition of QoL leads to a variety of instruments that are proposed to measure QoL [4]. This also applies for the measurement of QoL in patients with cochlear implantation since various QoL instruments have been used to evaluate the improvement of cochlear implantation and changes over time. Its measurement can serve as an addition to audiological performance and can provide a determining factor to perform systematic comparisons between groups, different treatment strategies and change over time (Table 1).

\section{ASSESSING QUALITY OF LIFE IN PATIENTS WITH COCHLEAR IMPLANT}

\section{Generic Instruments}

Generic instruments have been developed to capture a wide range of aspects in the health status of a person [5]. These instruments are not disease-specific, so they can be used in various different health problems. Even a "normative value" of the general population can be generated. An advantage of generic instruments is the comparability of different groups, treatments or a comparison with scores of the general population. As they cover a broad range of health problems, they may include items, that are not relevant for a 
Table 1. Improvements in QoL after Cochlear Implantation



specific disease or treatment and therefore they tend to be less sensitive to changes in a relevant aspect [5].

The most widely used generic instrument for assessing health-related quality of life is the Short-Form Health Survey (SF-36) questionnaire [6]. It quantifies the health status in eight dimensions: physical functioning, role limitations due to physical problems, role limitations due to emotional problems, social functioning, mental health, energy/vitality and general perception of health [4].

The SF-12 is a shortened version of the SF-36, it contains questions of all eight dimensions and has been proved to be reliable, valid and sensitive to changes [7].

Both questionnaires, the SF-36 and the SF-12, served as a co-instrument in various reports about the change of QoL in cochlear implantation.

The Glasgow Benefit Inventory (GBI) is a generic quality of life questionnaire that has been developed to detect the change in health status after otorhinolaryngological procedures [8]. Its validity has been proved for various interventions [9-11]. In contrast to this change questionnaire a current state measure was developed, the Glasgow Health Status Inventory (GHSI), which measures the effect of a health problem on the quality of life of a person. Both questionnaires use three different subscales to assess the change of health status: a generic subscale (12 questions), a social support subscale and a physical health subscale (each three questions) [12].
The Hearing Participation Scale (HPS) [13] is a shortened form of the Glasgow Health Status Inventory with 11 items that cover the three dimensions self-esteem related to hearing, level of social interaction related to hearing and hearing handicap $[14,15]$. Validity, reliability and sensitivity have been proved for the HPS by Hawthorne and Hogan in 2002 [13].

Utility measures are developed to provide information of a health status and quality of life by producing utility scores. They have been created to provide cost and utility for different health care interventions and may be useful in economic evaluation of competing interventions. In contrast to generic instruments they focus more on the overall score of the respondent than on scores of different dimensions of the health status. Usually there is no simple sum score of all items, each item has a different weight in the total score. A familiar example of a utility index is the Health Utilities Index (HUI). In this case, "utility" has rather the meaning of "preference" than "usefulness" [16]. The health state of the individual respondent can be estimated by preferences of the general population for different health states. The score for each health state is calculated from valuation by samples of the general population [5]. This particular scoring system provides utility (preference) scores on a generic scale, perfect health equals 1.00 and dead equals 0.00 . Curiously a score below zero can be admitted and corresponds to states worse than death [16]. Eight health status attributes are measured: vision, hearing, speech, ambulation, dexterity, 
emotion, cognition, self-care and pain [17]. The HUI exists in three versions, the HUI-2 and the HUI-3 are commonly used. Their reliability and validity has been proven in a wide variety of clinical studies [18].

The Assessment of Quality of Life (AQoL) is another health utility questionnaire with its five dimensions illness, independent living, social relationships, physical senses, and psychological wellbeing $[19,20]$. AQoL has proved to be a valid, reliable and sensitive instrument $[19,20]$.

Since utility measures are similar to generic health status measures some authors consider this type of health status evaluation not as a distinct class of measure but more as a generic health status measure with a particular form of numerical weighting or validation of health states $[5,17]$.

\section{Disease Specific Instruments}

Besides generic instruments disease specific instruments are intended to provide the patients' perception of a specific disease or health problem [5]. The items have been developed to assess the particular health problem, so the content is strictly relevant when used for a specific disease. Furthermore these instruments are more sensitive to detect changes over time in the particular disease. A major disadvantage is the incomparability. As it focuses on various problems arising with a certain condition, it is not possible to perform this questionnaire on a group without this certain condition. Consequentially a "normative value" of the general population cannot be generated.

Examples of hearing related specific instruments are the Patient Quality of Life Form (PQLF), the Index Relative Questionnaire Form (IRQF), the Performance Inventory for Profound Hearing Loss Answer Form (PIPHL) and the Nijmegen Cochlear Implant Questionnaire (NCIQ).

The Patient Quality of Life Form (PQLF) and the Index Relative Questionnaire Form (IRQF) were developed at the House Ear Institute Los Angeles [21] to provide information about the change of QoL after Cochlear implantation. The PQLF contains 38 questions covering the patients' coping with the hearing loss and five additional questions concerning the adaptation and changes noticed after the implantation. Eight major topics were included in the PQLF: sense of safety, emotional reactions, nature of interpersonal relationships, social activities, sense of isolation, communication problems, employment and recreational activities [21].

The respondent of the Index Relative Questionnaire Form (IRQF) is a near relative of the patient. It contains 31 questions and provides information about the relative's experience about the effect of the hearing impairment on the patient's lifestyle and daily activities as well as the patient's adaptation to the implant [21]. Both questionnaires have been validated and shown to be reliable.

The PIPHL assesses the patients' perception by 20 questions about daily situations regarding communication abilities [22]. It can also be used for hearing impaired patients.

Hinderink, et al. introduced a specific QoL instrument for users of the cochlear implant. The authors argue, that the PQLF only encompasses the psychological and social domain, but lacks a physical component [23]. Furthermore the original questionnaire does not have general domains or subdomains, although a factor analysis was performed by Mo, et al. in the meantime [24], six categories in the PQLF and five categories in the IRQF were identified. Hinderink et al. [23] introduced a novel questionnaire specific for cochlear implant users, the Nijmegen Cochlear Implantation Questionnaire (NCIQ). Three general domains were distinguished: physical functioning (with subdomains "sound perception basic", "sound perception advanced" and "speech production"), psychological functioning (with the subdomain "self-esteem") and social functioning (with the subdomains "activity" and "social interaction").

The NCIQ has become a standard questionnaire in assessing the QoL of patients with cochlear implants, has been validated and shown to be reliable and sensitive to clinical changes [23, 25-29].

\section{IMPACT OF COCHLEAR IMPLANTATION IN GENERIC QUALITY OF LIFE INSTRUMENTS AND UTILITIES}

\section{The SF-36 and the Health Utilities Index}

Krabbe, Hinderink and van de Broek investigated 45 postlingually deafened adult multichannel users in terms of SF-36 and HUI-2 before and after implantation. Significant improvements were found in five of seven domains of the SF-36 and two of six domains in the HUI-2 after implantation. The preimplant survey was obtained retrospectively (after implantation) but preimplant scores corresponded well with a control group of 46 patients on the waiting list for a cochlear implant. However, significant differences between the candidates of the waiting list and the cochlear implant recipients could be measured only in two domains of the SF-36 [25].

Six years later, Damen, et al. reinvestigated the group of CI patients recruited by Hinderink and Krabbe [28]. In the SF-36 a significant decrease in five of eight domains was found in the cochlear implant group after six years. In the former control group 22 of 46 patients had been implanted within six years and a significant increase in the SF-36 score was only found in the domain "mental health". Because of the ambiguous results of the SF-36 score the authors conclude, that the SF-36 should not be the first choice of generic QoL questionnaire when evaluating hearingimpaired patients [28].

In accordance to these results Mo, et al. found a significant difference only in one domain ("mental health") of the SF-36 between 84 users of a cochlear implant and 19 accepted but not operated candidates. Furthermore the authors found scores of both groups on the same level as the Norwegian general population. Their data suggest generic QoL measures like the SF-36 are of limited value in the evaluation of patients with hearing impairment [24].

The same authors performed a prospective study with 27 postlingually deafened patients and used the SF-36 before and 12-15 months after implantation. A significant difference was found only in the domain "general health" but not in the other domains. They endorsed their opinion about 
the SF-36 as not being suitable to detect changes in QoL of cochlear implant recipients [30].

In the report of Hirschfelder, et al. an investigation of 56 adult postlingually deafened patients was performed before and after implantation. Scores of the SF-36 improved significantly in four domains, but decreased significantly in two other domains after implantation [27].

In another report Damen, et al. did not find a significant difference between the score of the SF-12 in Usher type I patients who had received a cochlear implant and those who had not. The authors suggest the benefit of cochlear implantation is limited to the specific hearing domains of quality-of-life questionnaires [29].

Bess reviewed the SF-36 in studies determining hearing aids benefit. The SF-36 did not seem sensitive enough for a use in the hearing impaired population. As some items of the SF-36 appear to be weighted in favour of the physical domain and are designed to be sensitive to chronic conditions affecting mobility but not for hearing impaired patients [31].

Although the SF-36 is commonly used as a standard instrument for assessing the quality-of-life, it does not seem sensitive enough to capture the change in the QoL after receiving a cochlear implant.

The accordance of SF-36 scores in cochlear implant users, patients on the waiting list for cochlear implantation and the Norwegian general population [24] is a strong argument against using the SF-36 to measure QoL in patients with cochlear implantation.

In the report of Damen et al. the score of the Health utility Index (HUI-3) was also studied before and after cochlear implantation of 22 postlingually deafened adult patients. They found a significant increase of total score and the domains "hearing" and "emotions" but not in the other six domains [28].

The total HUI-2 score of eight prelingually deafened adults increased significantly five months after implantation, but this was due to the disproportional increase in the domain "sensation" (including the subdomains "hearing", "vision" and "speech"). Other domains did not change significantly as described by Klop et al. [32].

Francis et al. [33] observed a significant increase in HUI3 score in 47 patients (pre- and postlingually deafened) and in the subcategories "hearing" and "emotion". When regarded separately, the postlingually deafened patients achieved an improvement superior to the prelingually deafened patients.

After cochlear implantation a significant change is mainly seen in the two subdomains "hearing" and "emotion", the score of other subdomains seem to remain steady.

\section{The Assessment of Quality of Life and the Hearing Participation Scale}

Hawthorne et al. evaluated the QoL in 34 patients before implantation and again at a three- and a 6-month follow-up by using the AQoL and the HPS questionnaire [14]. A differentiation between pre- and postlingually deafened patients was not performed. The AQoL and the HPS score improved significantly from preimplantation to 3 months postimplantation and again to 6 months postimplantation. A comparison between general healthy population and hospital outpatients was also itemized. The preimplant score and the 3-month follow-up score were found to be well below AQoL norms for the general healthy population as well as for hospital outpatients. But at the 6-month follow-up the AQoL score converged with the norm for hospital outpatients. Further on an interesting analysis of the data in terms of the socioeconomic status of the patients and outcome after implantation was performed. In all groups preimplant scores and 3-month follow-up scores in the $\mathrm{AQoL}$ were significantly below the AQoL population norm. At the 6month follow-up only the patients with high socioeconomic status obtained scores similar to the population norm, whereas the patients with low and middle socioeconomic status did not. The authors argue, that some patients may be constrained by low socioeconomic status and they may require different rehabilitation needs. Interventions have to be adapted to meet such needs [14].

Since there was a significant change in AQoL and HPS score between the 3-month follow-up and the 6-month follow-up it would have been of interest how patients would perform in a long term follow-up past the six months after implantation. Other authors report a steady state in QoL as recently as up to three years after implantation [34].

In another study, Hogan and Hawthorne compared the QoL of 148 adult patients with cochlear implant to 54 deafened persons without a cochlear implant. Again they used the HPS and the AQoL questionnaire as QoL instruments. In both instruments users of cochlear implant scored far better than the non-users. In the HPS scale the total score and all three subscales ("self-esteem related to hearing", "level of social interaction related to hearing" and "hearing handicap") were significantly better, whereas in the AQoL questionnaire the total score was significantly higher, but only one subscale ("physical senses") differed significantly [15].

The HPS and the total score of AQoL seem promising instruments, both questionnaires have proven to be reliable and sensitive generic utility measures. Since they have not been used commonly in patients with hearing impairment, further studies are needed to affirm the data provided by Hawthorne et al.

\section{IMPACT OF COCHLEAR IMPLANTATION IN DISEASE SPECIFIC QUALITY OF LIFE INSTRUMENTS}

\section{The Patients Quality Life Form and the Index Relative Questionnaire Form}

In 1982 Wexler et al. developed preimplant and postimplant questionnaires to evaluate the value of the cochlear implant patient as being judged by the patient and by an individual in close relationship to the patient. So the Patients Quality Life Form (PQLF) and the Index Relative Questionnaire Form (IRQF) were devised. After implantation the patients had improvements in terms of "communication", "feeling of isolation", "feeling of being a 
burden to others", "concern for safety", "self-sufficiency" and "comfort at social events". The IRQF was consistent with the patients' perceptions [21].

Maillet, Tyler and Jordan used the PQLF and the IRQF in a study with 71 cochlear implant users. Compared to preimplant scores $48 \%$ of the patients had a higher score 24 months thereafter. The increase of PQLF scores was significant. Consistent with the PQLF data, $58 \%$ of the patients' relatives felt the cochlear implant had a positive impact on the patients' life, the overall score of the IRQF was significantly higher than the preimplant score [35]. Unfortunately the study does not differentiate between preand postlingually deafened patients, as this point may also impact the improvement of QoL by cochlear implantation $[32,33]$.

Mo et al. did not find a significant difference in the total PQLF score between 84 users of a cochlear implant and 19 accepted but not operated candidates for a cochlear implant. They performed a factor analysis within the PQLF and identified six categories. 1) "relations to close individuals", 2) "how communication and hearing affects life", 3) "work", 4) "hobbies", 5) "isolation and relations to friends" and 6) "feeling of being a burden and of belonging". A significant difference between these two groups was found in the two categories "how communication and hearing affects life" and "feelings of being a burden and of belonging". A non significant difference was seen in the category "isolation and relations to friends". The IQRF factor analysis revealed five content areas, the total score and the category "communication" were significantly higher for the patient group with the cochlear implant [24].

In a prospective study with 27 postlingually deafened patients the total score of PQLF and IRQF showed a significant improvement $12-15$ months after implantation compared to the scores obtained before implantation. The largest improvement was seen in the subcategories "how communication and hearing affects life" and "feelings of being a burden and belonging". Only two of the six categories did not show a significant improvement. Consistent with the PQLF scores, the total scores of the IRQF also gained significantly after implantation, so did all except one category within the IRQF. The most considerable improvement was seen in the category "communication" [30].

The PQLF and the IRQF seem to be a reliable, valid and sensitive instrument in assessing the QoL of patients with cochlear implant. The factor analysis performed by Mo et al. [24] allows a better differentiation within the QoL scores and hence information about changes in different aspects of daily life can be evaluated.

\section{The Nijmegen Cochlear Implantation Questionnaire}

Hinderink [23] and Krabbe [25] introduced a quantifiable disease specific health-related QoL instrument with a questionnaire on postlingually deafened patients. The scores of 45 patients after cochlear implantation were measured by the NCIQ questionnaire. Both scores, the pre- and the postimplant score, were assessed retrospectively after the implantation. As a control group the scores of 46 accepted but not operated patients on the waiting list for a cochlear implant were used. The scores of all six subdomains ("basic sound perception", "advanced sound perception", "speech production", "self-esteem", "activity" and "social interactions") significantly improved in the postimplant scores. Also a significant increase compared to the score of the control group could be examined. The most relevant differences were seen in the subdomains "basic" and "advanced sound perception". Still no obvious correlation between sound reception test and any of the 6 subdomains could be found.

In a long-term follow-up 6 years after the implantation 37 patients in the report of Hinderink and Krabbe were reinvestigated by Damen et al. [28]. By and large the NCIQ scores of the implanted patients stayed constant in all subdomains except the "social interactions" subdomain, where a significant decrease could be recognized. Still the beneficial effect of the cochlear implant remained clearly visible. However a small but non significant trend to deterioration was seen in most of the subdomains. As this effect could also be seen in a control group who did not receive a cochlear implant the authors contribute this deterioration to a natural effect of aging. In contrast to the reports of Hinderink [23] and Krabbe [25] the mean scores of the NCIQ score correlated well with a syllable and a phoneme speech test score. 22 patients of the non-cochlear implant control group mentioned in the report of Hinderink [23] and Krabbe [25] had been provided with cochlear implant systems in the meantime. In comparison to their preimplant NCIQ scores a significant improvement could be found after implantation. The extent of the improvement was similar to the improvement of scores found by Krabbe [25] and Hinderink [23] after implantation.

In a similar survey of Hirschfelder et al. [27] the NCIQ scores of 56 adult, postlingually deafened patients were determined. As in the survey of Krabbe [25] and Hinderink [23] both scores, the pre- and the postimplant score were assessed retrospectively. A significant increase was seen in all subdomain scores and in the total score after cochlear implantation. The largest differences were seen in the subdomains "basic sound perception" and "advanced sound perception". In accordance to the results of Damen et al. [28] significant correlations could be detected between postimplant scores (total scores and scores in the subdomains "advanced sound perception" and "speech production") and results in speech perception tests. No association was found between NCIQ scores and length of hearing loss or current age or age at implantation. In contrast, the duration of deafness had a strong influence on postimplant speech tests.

Cohen et al. [26] investigated the NCIQ scores of 24 postlingually deafened users of a cochlear implant and 27 users of a hearing aid. Scores were collected again retrospectively for the period prior and after the patients began the use of either cochlear implants or hearing aids. Both groups perceived benefits from their particular auditory intervention. As patients with a hearing aid had higher initial scores than patients with cochlear implants, patients with a cochlear implant had twice as much improvement than the hearing aids patients. Increased scores in the NCIQ total score and all subdomains were found among users of the cochlear implant and users of hearing aids. Furthermore a 
correlation between the NCIQ score and the audiological improvement as seen in speech perception tests was found.

In two other reports the NCIQ was used in particular groups of patients: Klop et al. investigated the outcome of QoL in eight adults with prelingual deafness [32]. NCIQ scores were gathered before implantation and at several points in time after implantation. They found a significant increase in the subdomains "sound perception basic", "sound perception advanced" and "social interaction" four months after implantation, but steady scores at twelve months and at 30 months after implantation.

Damen et al. performed a comparison of the NCIQ scores of patients with Usher Syndrome Type I with and without a cochlear implant [29]. The subdomains "sound perception basic" and "advanced" indicated significantly better results in adults and children equipped with a cochlear implant. Additionally children with a cochlear implant scored significantly better in the subdomains "activity" and "social interactions" than unimplanted children. As a lifestyle questionnaire specific for Usher Syndrome indicates, users of the cochlear implant need less help from others and remain more independent in daily life. It is concluded, that the QoL in multiply handicapped patients like patients with Usher Syndrome Type I can be enhanced by the use of a cochlear implant. Distinct differences could be seen especially in hearing-related quality of life items.

The NCIQ provides a sensitive and reliable instrument to rate the quality-of-life in patients provided with a cochlear implant. The questionnaire is able to detect a wide variety of aspects within the QoL. In most of the reports significant improvements in the NCIQ scores were observed in total scores as well as in all subdomains [23, 26-28]. In other small scale studies at least the subdomains "sound perception basic" and "advanced" changed significantly [29, 32] as these two subdomains seem to be the most susceptible alterations affected by cochlear implantation. The NCIQ should be used as a first-line questionnaire in future studies to monitor technical innovations and rehab strategies. A minor flaw in the establishment of the NCIQ in the above mentioned reports is the retrospective collection of preimplantation scores [23, 26, 27]. Even though reports indicate that retrospectively collected preimplantation data was comparable to data of patients on a waiting list for a cochlear implant [23] or similar to prospective data [28], other authors did not revalidate the comparability between these groups with self-provided data.

\section{Relationship Between QoL Scores and Speech Perception Tests}

Several studies suggest a correlation between scores in QoL questionnaires and the outcome in speech perception tests. Cohen et al. found a correlation between the improvement in total NCIQ score and sentence recognition scores. This finding is consistent with those of Francis et al. [33], Vermeire et al. [36] and Hirschfelder et al. [27] who described a similar correlation between speech perception tests and QoL scores. However, several other studies could not confirm this correlation [23, 30, 35, 37]. It is argued, that the patients subjective perception is not directly linked to objective hearing tests [23]. This is supported by the fact, that prelingually deafened adults report a noticeable improvement in the QoL scores although only a modest increase in objective hearing test is achieved [38]. Hirschfelder et al. only found a significant correlation between two subdomains ("sound perception basic" and "advanced") and speech perception tests but substantial increase in all subdomains [27]. The authors argue that the effect of cochlear implantation on QoL might even outweigh the improvements in hearing that could be detected in speech perception tests.

\section{Relationships Between QOL Scores and Duration of Deafness Before Implantation}

Maillet et al. found a significant correlation between duration of deafness and differences in QoL scores. The authors found a significant correlation between the degree of improvement and the number of years the patient had been deaf: patients, who were deaf for 10 years or less showed a greater improvement in PQLF scores than patients who had been deaf for 20 years or longer [35]. However various other studies were unable to confirm these results $[14,26,27,30]$. Still, there is good evidence that the duration before implantation influences audiological performance after implantation: longer duration of deafness correlates with lower scores in speech tests results $[27,39]$.

Compared to speech and hearing tests QoL scores are able to capture a broader view on the patients' daily life, in this respect QoL scores do not necessarily coincide with scores of hearing tests. Correlations found in hearing tests cannot be transferred to correlations in QoL scores.

\section{QoL Scores and the Duration of the Cochlear Implant Use}

The duration of the use of the cochlear implant may influence the QoL scores. Results of different studies are inconclusive. Hirschfelder et al. detected a positive correlation between time since intervention and NCIQ scores [27]. These results are in contrast to the report of Damen et al. who even found a trend to deterioration in NCIQ scores in a time period of six years [28]. This deterioration could be a natural effect of aging since a control group without a cochlear implant showed a similar trend in NCIQ scores. Hence this descent of NCIQ scores might not be related to cochlear implantation.

\section{Improvement in QoL in Elderly Candidates}

Cochlear implantation in the elderly is under discussion, because it is assumed that the improvement in elderly patients might be poor due to limited physical and cognitive capabilities as well as otologic factors [40]. Consequentially the improvement of QoL is of interest to validate the effect of cochlear implant on the elderly patient's daily life. Vermeire et al. investigated 89 postlingually deafened patients [36]. For the definition of different age groups, the cohort was divided into a younger age (below 55 years), middle aged (56 to 69 years) and a geriatric group (70 years or older). All groups showed a significant improvement after implantation in the score of the Glasgow Benefit Inventory. A significant difference between the age groups could not be seen. Other studies have noted a similar increase in QoL score $[33,41,42]$. In conclusion older age does not seem to 
have an impact on QoL scores. Older patients see a similar improvement in their daily life activities as middle aged patients.

\section{CONCLUSION}

Numerous instruments to assess the QoL in patients with a cochlear implant have been developed and validated in the past. Several reports prove the outstanding impact of cochlear implantation on the patients' daily life activities as it is seen in the improvement in scores of several QoL instruments. Remarkable improvements can be seen in several aspects of QoL, including physical, social and psychological domains. Generic instruments like the SF-36 may focus on the physical domain and are designed to be sensitive to chronic diseases and focus on conditions that may not be influenced by cochlear implantation such as mobility. So its use may not result in sensitive findings [31]. Still certain subdomains of the SF-36 show considerable improvements. When comparing these subdomains, the degree of improvement achieved by cochlear implantation is even comparable to that of renal or heart transplantation [25].

In the literature authors focused either on the NCIQ or on PQLF and IRQF as disease specific instruments. Both approaches have been proved to be reliable and valid, but interestingly a direct comparison between NCIQ and PQLF/IRQF has not been performed by now.

QoL instruments are an essential addition to speech perception tests to quantify the outcome of cochlear implants. Compared to speech perception tests QoL scores allow a more comprehensive insight into patients' daily life and activities. Cochlear implant related QoL states an important instrument to provide information about the outcome of technical improvements, different treatment and rehab strategies in future.

\section{REFERENCES}

[1] Clark G. Cochlear implants fundamentals and applications. New York: Springer 2003.

[2] Fetterman BL, Domico EH. Speech recognition in background noise of cochlear implant patients. Otolaryngol Head Neck Surg 2002; 126(3): 257-63.

[3] Hamzavi J, Franz P, Baumgartner WD, Gstoettner W. Hearing performance in noise of cochlear implant patients versus severelyprofoundly hearing-impaired patients with hearing aids. Audiology 2001; 40(1): 26-31.

[4] Sanders C, Egger M, Donovan J, Tallon D, Frankel S. Reporting on quality of life in randomised controlled trials: bibliographic study. BMJ (Clinical research ed) 1998 31; 317(7167): 1191-4.

[5] Fitzpatrick R, Davey C, Buxton MJ, Jones DR. Evaluating patientbased outcome measures for use in clinical trials. Health Technol Assess (Winchester, England) 1998; 2(14): i-iv, 1-74.

[6] Ware JE, Jr., Sherbourne CD. The MOS 36-item short-form health survey (SF-36). I. Conceptual framework and item selection. Med Care 1992; 30(6): 473-83.

[7] Ware J, Jr., Kosinski M, Keller SD. A 12-Item Short-Form Health Survey: construction of scales and preliminary tests of reliability and validity. Med Care 1996; 34(3): 220-33.

[8] Robinson K, Gatehouse S, Browning GG. Measuring patient benefit from otorhinolaryngological surgery and therapy. Ann Otol Rhinol Laryngol 1996; 105(6): 415-22.

[9] Bhattacharyya N, Kepnes LJ. Economic benefit of tonsillectomy in adults with chronic tonsillitis. Ann Otol Rhinol Laryngol 2002; 111(11): 983-8.
[10] Fahy C, Nikolopoulos TP, O'Donoghue GM. Acoustic neuroma surgery and tinnitus. Eur Arch Otorhinolaryngol 2002; 259(6): 299301.

[11] McKiernan DC, Banfield G, Kumar R, Hinton AE. Patient benefit from functional and cosmetic rhinoplasty. Clin Otolaryngol Allied Sci 2001; 26(1): 50-2.

[12] Institute of hearing research. The Glasgow Health Status Questionnaires Manual 1998; Available from: http://www.ihr.mrc. ac.uk

[13] Hawthorne G, Hogan A. Measuring disability-specific patient benefit in cochlear implant programs: developing a short form of the Glasgow Health Status Inventory, the Hearing Participation Scale. Int J Audiol 2002; 41(8): 535-44.

[14] Hawthorne G, Hogan A, Giles E, et al. Evaluating the healthrelated quality of life effects of cochlear implants: a prospective study of an adult cochlear implant program. Int J Audiol 2004; 43(4): 183-92.

[15] Hogan A, Hawthorne G, Kethel L, et al. Health-related quality-oflife outcomes from adult cochlear implantation: a cross-sectional survey. UK Cochlear Implants Int. 2001; 2(2): 115-28.

[16] UK Cochlear Implant Study Group. Criteria of candidacy for unilateral cochlear implantation in postlingually deafened adults I: theory and measures of effectiveness. Ear Hear 2004; 25(4): 31035 .

[17] Torrance GW, Furlong W, Feeny D, Boyle M. Multi-attribute preference functions. Health Utilities Index. PharmacoEconomics 1995; 7(6): 503-20.

[18] Furlong WJ, Feeny DH, Torrance GW, Barr RD. The Health Utilities Index (HUI) system for assessing health-related quality of life in clinical studies. Ann Med 2001; 33(5): 375-84.

[19] Hawthorne G, Richardson J, Osborne R. The Assessment of Quality of Life (AQoL) instrument: a psychometric measure of health-related quality of life. Qual Life Res 1999; 8(3): 209-24.

[20] Hawthorne G, Richardson J, Day NA. A comparison of the Assessment of Quality of Life (AQoL) with four other generic utility instruments. Ann Med 2001; 33(5): 358-70.

[21] Wexler M, Miller LW, Berliner KI, Crary WG. Psychological effects of cochlear implant: patient and "index relative" perceptions. Ann Otol Rhinol Laryngol 1982; 91(2 Pt 3): 59-61.

[22] Owens E, Raggio M. Performance inventory for profound and severe loss (PIPSL). J Speech Hear Disord 1988; 53(1): 42-56.

[23] Hinderink JB, Krabbe PF, Van Den Broek P. Development and application of a health-related quality-of-life instrument for adults with cochlear implants: the nijmegen cochlear implant questionnaire. Otolaryngol Head Neck Surg 2000; 123(6): 756-65.

[24] Mo B, Harris S, Lindbaek M. Cochlear implants and health status: a comparison with other hearing-impaired patients. Ann Otol Rhinol Laryngol 2004; 113(11): 914-21.

[25] Krabbe PF, Hinderink JB, van den Broek P. The effect of cochlear implant use in postlingually deaf adults. Int $\mathrm{J}$ Technol Assess Health Care 2000; 16(3): 864-73.

[26] Cohen SM, Labadie RF, Dietrich MS, Haynes DS. Quality of life in hearing-impaired adults: the role of cochlear implants and hearing aids. Otolaryngol Head Neck Surg 2004; 131(4): 413-22.

[27] Hirschfelder A, Grabel S, Olze H. The impact of cochlear implantation on quality of life: the role of audiologic performance and variables. Otolaryngol Head Neck Surg 2008; 138(3): 357-62.

[28] Damen GW, Beynon AJ, Krabbe PF, Mulder JJ, Mylanus EA. Cochlear implantation and quality of life in postlingually deaf adults: long-term follow-up. Otolaryngol Head Neck Surg 2007; 136(4): 597-604.

[29] Damen GW, Pennings RJ, Snik AF, Mylanus EA. Quality of life and cochlear implantation in Usher syndrome type I. Laryngoscope 2006; 116(5): 723-8.

[30] Mo B, Lindbaek M, Harris S. Cochlear implants and quality of life: a prospective study. Ear Hear 2005; 26(2): 186-94.

[31] Bess FH. The role of generic health-related quality of life measures in establishing audiological rehabilitation outcomes. Ear Hear 2000; 21(4 Suppl): 74S-9S.

[32] Klop WM, Briaire JJ, Stiggelbout AM, Frijns JH. Cochlear implant outcomes and quality of life in adults with prelingual deafness. Laryngoscope 2007; 117(11): 1982-7.

[33] Francis HW, Chee N, Yeagle J, Cheng A, Niparko JK. Impact of cochlear implants on the functional health status of older adults. Laryngoscope 2002; 112(8 Pt 1): 1482-8. 
[34] Zhao F, Bai Z, Stephens D. The relationship between changes in self-rated quality of life after cochlear implantation and changes in individual complaints. Clin Otolaryngol 2008; 33(5): 427-34.

[35] Maillet CJ, Tyler RS, Jordan HN. Change in the quality of life of adult cochlear implant patients. Ann Otol Rhinol Laryngol 1995; 165: 31-48.

[36] Vermeire K, Brokx JP, Wuyts FL, Cochet E, Hofkens A, Van de Heyning PH. Quality-of-life benefit from cochlear implantation in the elderly. Otol Neurotol 2005; 26(2): 188-95.

[37] Kou BS, Shipp DB, Nedzelski JM. Subjective benefits reported by adult Nucleus 22-channel cochlear implant users. J Otolaryngol 1994; 23(1): 8-14

[38] Hinderink JB, Mens LH, Brokx JP, van den Broek P. Performance of prelingually and postlingually deaf patients using single-channel or multichannel cochlear implants. Laryngoscope 1995; 105(6): 618-22.

[39] Tyler RS, Summerfield AQ. Cochlear implantation: relationships with research on auditory deprivation and acclimatization. Ear Hear 1996; 17(3 Suppl): 38S-50S.

[40] Buchman CA, Fucci MJ, Luxford WM. Cochlear implants in the geriatric population: benefits outweigh risks. Ear Nose Throat 1999; 78(7): 489-94.

[41] Orabi AA, Mawman D, Al-Zoubi F, Saeed SR, Ramsden RT. Cochlear implant outcomes and quality of life in the elderly: Manchester experience over 13 years. Clin Otolaryngol 2006; 31(2): 116-22.

[42] Djalilian HR, King TA, Smith SL, Levine SC. Cochlear implantation in the elderly: results and quality-of-life assessment. Ann Otol Rhinol Laryngol 2002; 111(10): 890-5.

This is an open access article licensed under the terms of the Creative Commons Attribution Non-Commercial License (http: //creativecommons.org/licenses/by$\mathrm{nc} / 3.0 /$ ) which permits unrestricted, non-commercial use, distribution and reproduction in any medium, provided the work is properly cited. 